\title{
Status \& Challenges of Tracker Design for FCC-hh
}

\author{
Zbynek DRASAL*† \\ CERN, Switzerland \\ E-mail: Zbynek.Drasal@cern.ch
}

A $100 \mathrm{TeV}$ proton collider represents a core aspect of the Future Circular Collider (FCC) study. An integral part of this project is the conceptual design of individual detector systems that can be operated under luminosities up to $3 \times 10^{35} \mathrm{~cm}^{-2} \mathrm{~s}^{-1}$. Compared to HL-LHC scenario, one of the key limitations in the design arises from an increased number of pile-up events $\mathrm{O}(1000)$, c.f. $\mathrm{O}(140)$ at HL-LHC, making both particle tracking and identification of vertices extremely challenging. This paper reviews the general ideas that conceptually drive the current tracker/vertex detector design for the FCC-hh (proton-proton). These include material budget, detector granularity, pattern recognition, primary vertexing/pile-up mitigation and occupancy/data rates. Finally, the limits of current tracker technologies and requirements on their future progress, i.e. the dedicated R\&D $\ddagger$ are discussed.

The 26th International Workshop on Vertex Detectors

10-15 September, 2017

Las Caldas, Asturias, Spain

\footnotetext{
* Speaker.

${ }^{\dagger}$ On behalf of the FCC-hh Detector Working Group

${ }^{\ddagger}$ Research and Development
} 


\section{Introduction}

With the discovery of the Higgs boson at the Large Hadron Collider (LHC) at CERN in July 2012 the particle physics community has significantly advanced their understanding of the Standard Model. Particularly, how the massive elementary particles acquire their mass through the Brout-Englert-Higgs mechanism. However, despite its huge success, the LHC machine is close to reaching its technical limits and thus, may not be able to address a new frontier of fundamental questions. For example, those related to the largely mysterious Higgs particle: its quantum nature (a substructure of the Higgs boson), dynamics of Electro-Weak Symmetry Breaking (Higgs selfcoupling), or more general ones, like the origin of matter etc. [1,2]. This all necessitates another leap in accelerator technology, to higher collision energies and higher luminosities. The higher centre-of-mass energy, coupled with the greater luminosity, will increase the discovery potential and simultaneously allow measurements to be made with unprecedented statistical precision.

Among the various accelerator options a proton-proton $(\mathrm{pp} / \mathrm{hh})$ collider operating at energies far beyond that of the LHC has emerged as an appealing option, including the Future Circular Collider (FCC) project promoted by CERN [3] or the SppC project promoted by IHEP in China [4]. Due to various limits of foreseeable technologies (16 T bending magnets, $\mathrm{RF}^{1}$ acceleration system, large-scale cryogenics etc.) and/or other constraints anticipated by the proposing research agencies (the size of a tunnel, cost etc.) $\sqrt{s}=100 \mathrm{TeV}$ represents a benchmark - an upper energy limit, which is being considered for such a collider [1].



Figure 1: FCC project promoted by CERN - a layout scheme [7].

Clearly, an upgrade to a high energy and high luminosity accelerator machine does not represent the only challenge. The other one, forming an integral part of such a project, is to design an appropriate particle detector system. On one hand, it has to represent a very general-purpose detector (c.f. ATLAS [5] or CMS [6]) whilst providing an unprecedented precision in momentum/energy resolution over a wide range of energies - from several $\mathrm{GeV}$ up to tens of $\mathrm{TeV}$. On the other hand, it has to withstand the challenging operating conditions of the accelerator machine. In the FCC-hh environment, the biggest challenges in detector design arise mainly from a large

\footnotetext{
${ }^{1}$ Radio Frequency
} 
number of pile-up events $\mathrm{O}(1000)$, from the expected huge data rates and from the requirements on the applied technologies, such as the radiation hardness of individual sub-detectors etc.

The collaborative effort within the FCC focuses on 3 different case studies: FCC-hh (pp collider), FCC-ee (electron-positron collider) and FCC-eh (asymmetric e-p collider). FCC-hh represents the main goal of the study and defines thus the overall infrastructure [7] (see a layout scheme of $100 \mathrm{TeV}$ pp collider in a $97.75 \mathrm{~km}$ tunnel in Fig. 1). FCC-ee is studied as a possible intermediate step, FCC-eh as an option. The first milestone for the study is to prepare a common Conceptual Design Report (CDR) for the European Strategy Update by end of 2018.

This paper reviews the key ideas that drive the current conceptual design of the Tracking detector system, envisaged to be operated under FCC-hh conditions.

\section{FCC-hh and Reference Detector Layout}

The FCC-hh machine is planned to be run in two phases. In phase 1 (baseline) the machine will be operated at peak luminosity $L_{\text {peak }}=5 \times 10^{34} \mathrm{~cm}^{-2} \mathrm{~s}^{-1}$ for the first 10 years. It will yield $2.5 \mathrm{ab}^{-1}$ of collected data per experiment. Subsequently, the FCC-hh will be operated in phase 2 (ultimate) at $L_{\text {peak }} \leq 30 \times 10^{34} \mathrm{~cm}^{-2} \mathrm{~s}^{-1}$ for the following 15 years, which will yield additional $15 \mathrm{ab}^{-1}$ per experiment. In total, after 25 years of operation, we estimate collecting $\mathrm{O}(20) \mathrm{ab}^{-1}$ of data for each experiment $[7,8]$.

In terms of minimum bias events, the FCC-hh is expected to be very similar to the LHC (or HL-LHC [9]), but with a few important exceptions. These exceptions make the detector design very challenging. They include a huge increase in pile-up per bunch crossing $\mathrm{O}(1000)$ (c.f. $\mathrm{O}(140)$ expected for HL-LHC), huge particle/data rates in all detector sub-systems (a big challenge for a trigger and read-out technology) and significantly higher radiation levels in the innermost and forward parts of the detector. The radiation levels are demonstrated in Fig. 2, showing $1 \mathrm{MeV}$ neutron equivalent ( $1 \mathrm{MeV}$ neq.) fluence for the reference detector layout after $30 \mathrm{ab}^{-1}$ [10].

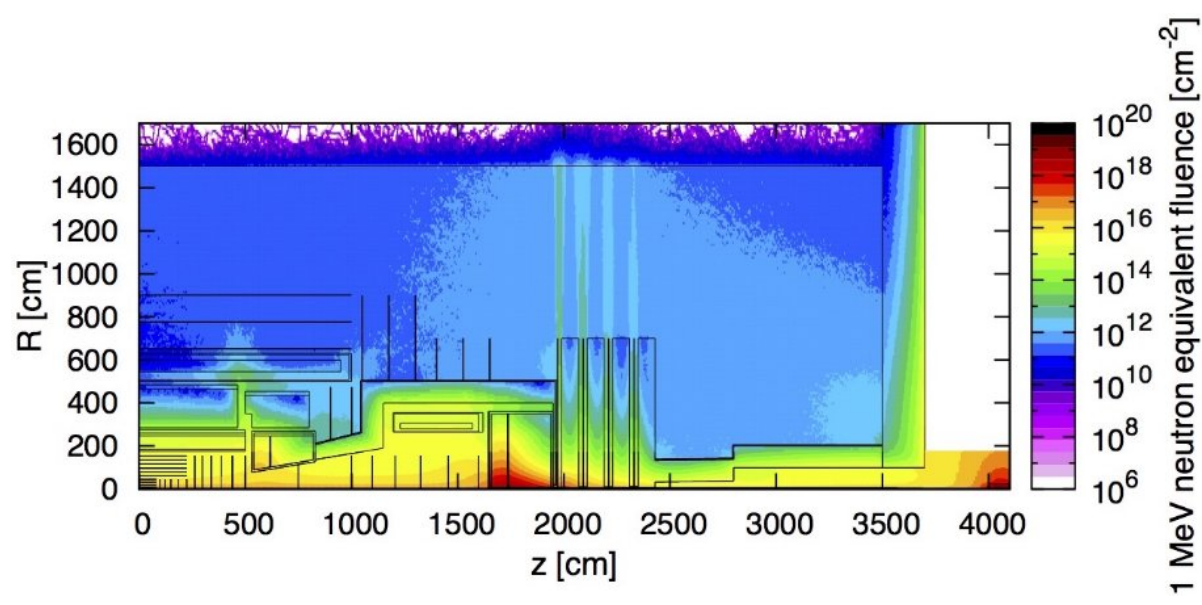

Figure 2: $1 \mathrm{MeV}$ neq. fluence for a reference detector layout after $30 \mathrm{ab}^{-1}$, as simulated by FCC-hh Fluka team [10].

From a detector point of view, the FCC-hh opens up a new kinematic and dynamical regime. In addition to the difficulty of measuring high $p_{\mathrm{T}}$ tracks or highly energetic jets, physicists will face 
the challenge of measuring highly collimated/boosted objects, particularly in the forward region. For details, follow for example the Higgs physics in a context of vector-boson fusion (VBF) or vector-boson scattering $[1,11]$. This all sets a strict requirement on the detector coverage in $\eta$ and the overall granularity of the detector. Generally, a highly granular detector with a precision tracking/calorimetry up-to $|\eta|=4.0$ is a must. Moreover, we require an extended acceptance (VBF measurements) up-to $|\eta|=6.0$ (c.f. ATLAS/CMS acceptance: $|\eta| \leq 2.5$ at LHC and $|\eta| \leq 4.0$ envisaged for HL-LHC). The final detector reference layout is depicted in Fig. 3.



Figure 3: The reference layout of FCC-hh detector [12, 13], consisting of a beryllium beam-pipe; central, intermediate (iFwd) and forward (FWD) tracker; Pb/LAr (lead/liquid argon) electromagnetic calorimeter; $\mathrm{Fe} / \mathrm{Sci}$ (Fe/scintillator) hadronic calorimeter in the barrel and $\mathrm{Pb} / \mathrm{LAr}$ in the forward region; muon system.

\section{Tracker - Design Driving Principles}

The tracking system is being designed with several constraints in mind. It has to provide a sufficient momentum resolution for high $p_{\mathrm{T}}$ particles whilst keeping a reasonable sensitivity for low $p_{\mathrm{T}}$ tracks. In addition, efficient vertexing and $b, c, \tau$-tagging are essential despite an extreme pile-up level. To fulfill such conditions, the tracker requires adequate bending power from the detector magnet system. The final goal is to achieve $\Delta p_{\mathrm{T}} / p_{\mathrm{T}}$ resolution at the level of 10-20\% at $p_{\mathrm{T}}=10 \mathrm{TeV} / \mathrm{c}$ (c.f. $10 \%$ at $p_{\mathrm{T}}=1 \mathrm{TeV} / \mathrm{c}$ for the current LHC experiments). In the last 2 years, several magnet scenarios have been studied [14]. The final reference design proposes a combination of a $4 \mathrm{~T}$ unshielded central solenoid ( $10 \mathrm{~m}$ free bore) and two $4 \mathrm{~T}$ unshielded solenoids in the forward regions. Applying a Gluckstern formula for such a setup:

$$
\Delta p_{\mathrm{T}} / p_{\mathrm{T}} \approx \frac{\sigma_{R \Phi} p_{\mathrm{T}}}{0.3 B L^{2}} f(N)
$$


one can derive the requirements for the intrinsic detector resolution in $R-\Phi, \sigma_{R \Phi} \approx 7.5-10 \mu \mathrm{m}$, and necessary tracker lever-arm, $L \approx 1.6 \mathrm{~m}$. Within the formula, the magnetic field, $B$, is assumed to be approximately constant, $B=4 \mathrm{~T}$. The factor, $f(N)$, corrects for the effects of layout geometry. Its value depends on number of layers $N$, their radii etc. To properly account for material effects, the formula needs to be extended to include multiple scattering (MS), especially for low $p_{\mathrm{T}}$ tracks.

There are several key parameters to be optimized for the tracker/vertex detector. Firstly, the granularity in $R-\Phi$. Besides its importance for the overall momentum resolution, one has to consider its importance on efficient tagging of displaced vertices via $\delta\left(d_{0}\right)$, i.e. the resolution of impact parameter in $R-\Phi$. Another requirement arises from a limit on detector occupancy $<1 \%$.

Secondly, the number of measurement layers, $N$. The increase in $N$ leads to an increase in the number of measurement points and a decrease in the propagation distance between individual hits. The latter is particularly important when connecting hits to a track candidate - a pattern-recogntion problem. In contrast, the higher $N$ leads to a bigger material budget, and thus, a larger measurement uncertainty arising from the MS. Generally, the effect of material increases approximately with increasing number of layers as $N$, the momentum resolution only as $1 / \sqrt{N}$. In the current design, we assume to have 4 pixel barrel (BRL) layers in the innermost region (for seeding purposes etc.), 4 macro-pixel BRL layers in the intermediate region (to fulfill the occupancy limit) and 4 BRL striplet/macro-pixel layers in the outermost region (to extend the lever-arm). A huge challenge for the technology of innermost layers will be a long term damage $\left(\approx 0.4 \mathrm{GGy}\right.$ after $30 \mathrm{ab}^{-1}$ at $r=25 \mathrm{~mm})$, and $1 \mathrm{MeV}$ neq. fluence $\left(\approx 6 \times 10^{17}\right.$ per $\mathrm{cm}^{2}$ at $\left.r=25 \mathrm{~mm}\right)$. These numbers are approximately 30 times (600 times) higher compared to HL-LHC (LHC) [10].
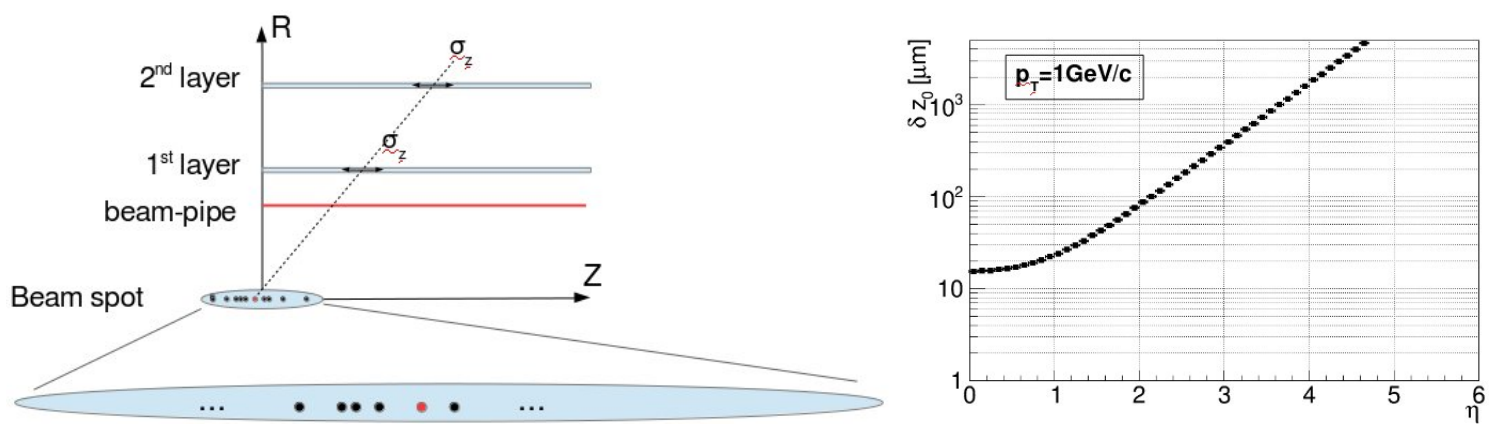

Figure 4: The pile-up mitigation scheme, illustrating an assignment of a given track to the correct primary vertex (left), and a natural limit on $\delta\left(z_{0}\right)$ for low $p_{\mathrm{T}}=1 \mathrm{GeV} / \mathrm{c}$ tracks due to a beam-pipe material (right).

Thirdly, the granularity in $Z$. One aspect of the optimization arises from the requirements on pattern-recognition and detector occupancy limit $<1 \%$. The other aspect is given by the need to mitigate the effect of pile-up events. The size of the luminous region (beam-spot) in $R-\Phi$ is approximately comparable to the detector resolution in $R-\Phi$. Hence, one can't expect to discriminate a signal primary vertex from other pile-up vertices $\mathrm{O}(1000)$ by using the track $\delta\left(d_{0}\right)$. In contrast, the luminous region in $Z$ is at the order of tens of millimeters. Assuming a scenario with a Gaussian profile of colliding bunches, with $\sigma_{z}^{\text {bunch }} \approx 75 \mathrm{~mm}$, and neglecting the effect of Piwinsky/Time-Piwinsky angles [15] here, the $\sigma_{z}^{\text {lum }}=\sigma_{z}^{\text {bunch }} / \sqrt{2} \approx 53 \mathrm{~mm}$. Clearly, the tracker with sufficient resolution in $Z$ may thus effectively help mitigate the pile-up effect.

Finally, assuming that a tracker provides timing information, in addition to the $Z$ measurement, 
the pile-up discrimination power opens up in both "dimensions": time and $Z$. The key question is whether and what timing resolution per track is needed for the FCC-hh scenario. To illustrate the whole problem see a sketch in Fig. 4. Notice that with an increasing track $\eta$, the limit on the $z_{0}$ resolution, $\delta\left(z_{0}\right)$, naturally appears due to MS in the cylindrical beam-pipe (BP). That's particularly limiting for the vertexing of low $p_{\mathrm{T}}$ particles.

\section{Reference Tracker Layout and Momentum Resolution}

The reference tracker (Fig. 5) extends in a radial direction from $20 \mathrm{~mm}$ (the beam-pipe radius) up to $1.55 \mathrm{~m}$, and in $Z$ up to approx. $\pm 16 \mathrm{~m}$. The large length in $Z$ is needed to cover the required $\eta$ acceptance, in combination with a sufficient $p_{\mathrm{T}}$ resolution and a minimum propagation distance between the measurement planes (an optimal pattern-recognition). As for the pattern-recognition, notice the specific design of 3 intermediate forward discs connecting the central and forward tracker in the end-cap (EC), and the design with tilted detectors in the BRL/EC transition area.
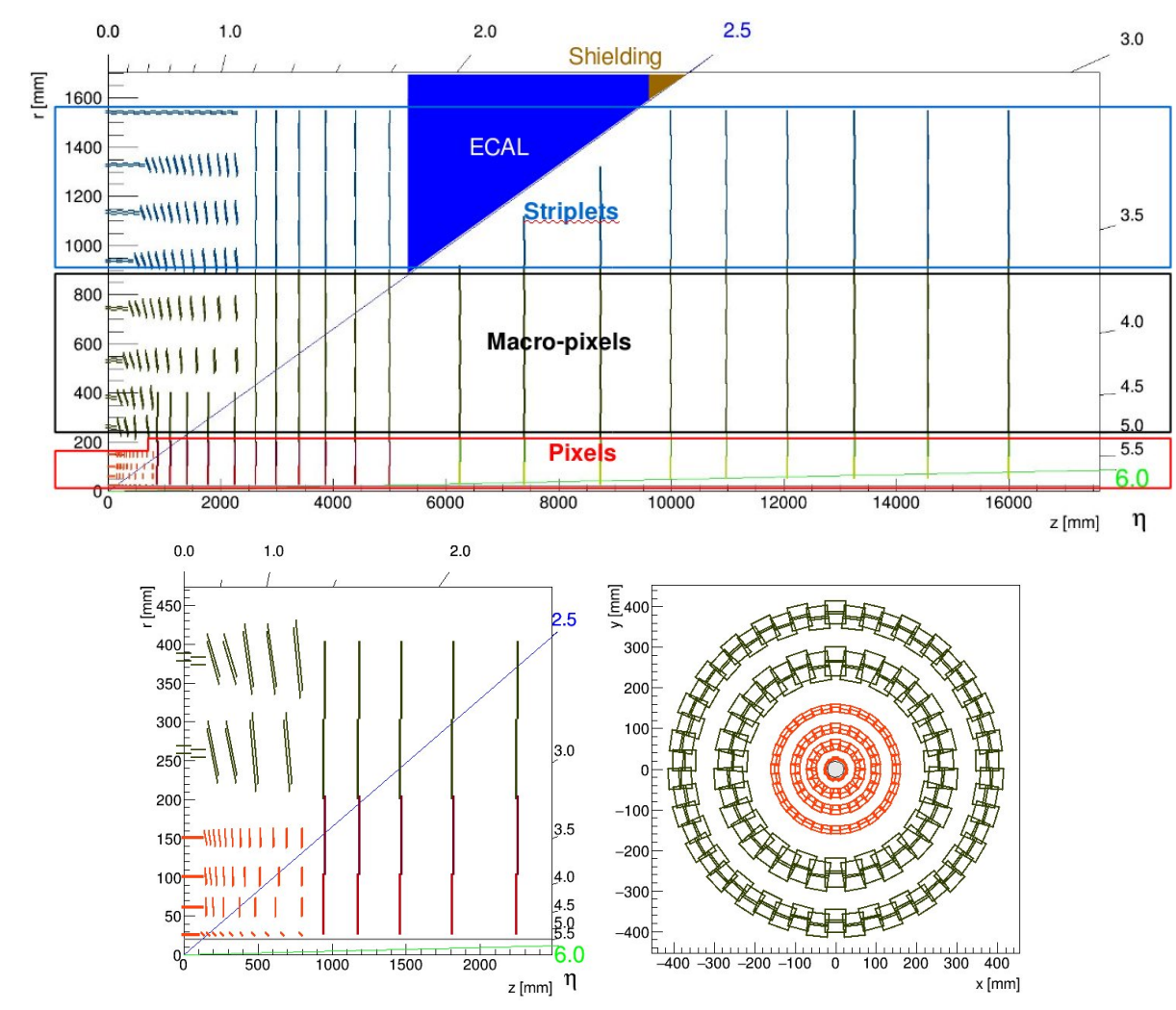

Figure 5: A reference tracker layout v4.01 as a result of the ongoing optimization - taking into account required $\eta$ coverage, $\Delta p_{\mathrm{T}} / p_{\mathrm{T}}$ resolution, pattern-recognition performance (notice the tilted layout), expected particle fluence (occupancy limit), optimal $\delta\left(z_{0}\right)$ etc. The $R-Z$ projection of the overall tracker is shown at the top. The detail of the pixel detector layout at the bottom: $R-Z$ projection on the left, $X-Y$ on the right.

The combined surface of tracker and vertex detector is approximately $391 \mathrm{~m}^{2}$. The summary of spatial dimensions of detector read-out cells for different categories of sensors is given in Table 1. Their values have been optimized to give as low a number of read-out $(r / o)$ channels as possible whilst fulfilling all the above criteria. Assuming a binary r/o, the number reaches nearly $17 \times 10^{9}$ 
channels: $4258 \times 10^{6}$ channels in the pixel region (red), $8734 \times 10^{6}$ in the macro-pixel region (black) and $3747 \times 10^{6}$ (blue) in the striplet region. In comparison to ATLAS or CMS, the huge increase in number of r/o channels is mainly due to an extreme tracker length along $Z$. (One needs to cover the full tracker length with pixel detectors out to a certain radius, due to occupancy limits, radiation level etc.) This opens up a natural question of choosing a suitable read-out technology and trigger design. If the untriggered mode is chosen, the read-out scheme would be challenged with the total tracker data rate of $\approx 650 \mathrm{~TB} / \mathrm{s}$. Other technology extremes arise from the anticipated data flows, which may reach several $\mathrm{Gb} / \mathrm{s} / \mathrm{cm}^{2}$ in the innermost 2 layers/rings.

\begin{tabular}{ccc}
\hline Pixels (inner) & Macro-pixels (middle) & Striplets/Macro-pixels (outer) \\
\hline $25 \times 50 \mu \mathrm{m}^{2}(1-4$ th BRL, 1st EC ring) & $33.3 \times 400 \mu \mathrm{m}^{2}$ & $33.3 \mu \mathrm{m} \times 1.75 \mathrm{~mm}$ (BRL) \\
$33.3 \times 100 \mu \mathrm{m}^{2}$ (2nd EC ring) & & $33.3 \mu \mathrm{m} \times 1.75 \mathrm{~mm}$ (EC) \\
$33.3 \times 400 \mu \mathrm{m}^{2}$ (3-4th EC ring) & $33.3 \mu \mathrm{m} \times 50 \mathrm{~mm}$ (12th BRL layer) \\
\hline
\end{tabular}

Table 1: The summary of spatial dimensions (pitch) of detector read-out cells for various regions.

The final question in the reference design concerns a realistic description of tracker material budget. The tracker itself opens many challenges for future technologies and thus, a dedicated $R \& D$ has to be still set up. In particular, due to extreme radiation levels the technologies for the innermost layers/rings remain completely open and the technologies applied within the LHC experiments (or their upgrades) can not be directly extrapolated. Therefore, a more pragmatic approach has to be used instead. We assume the following material composition of individual detectors: $20 \% \mathrm{Si}, 42 \%$ $\mathrm{C}, 2 \% \mathrm{Cu}, 6 \% \mathrm{Al}$ and $30 \%$ plastic. To account for an accumulation effect of services, cooling etc., the $x / x_{0}$ quantity is increased in steps with an increasing radius ( $x$, resp. $x_{0}$, stands for the material thickness, resp. radiation length, referenced to a perpendicular particle passage). Under such assumptions on material budget, the expected momentum resolution, as simulated using tkLayout software (SW), is shown in Fig. 6. The tkLayout SW tool was originally developed for CMS
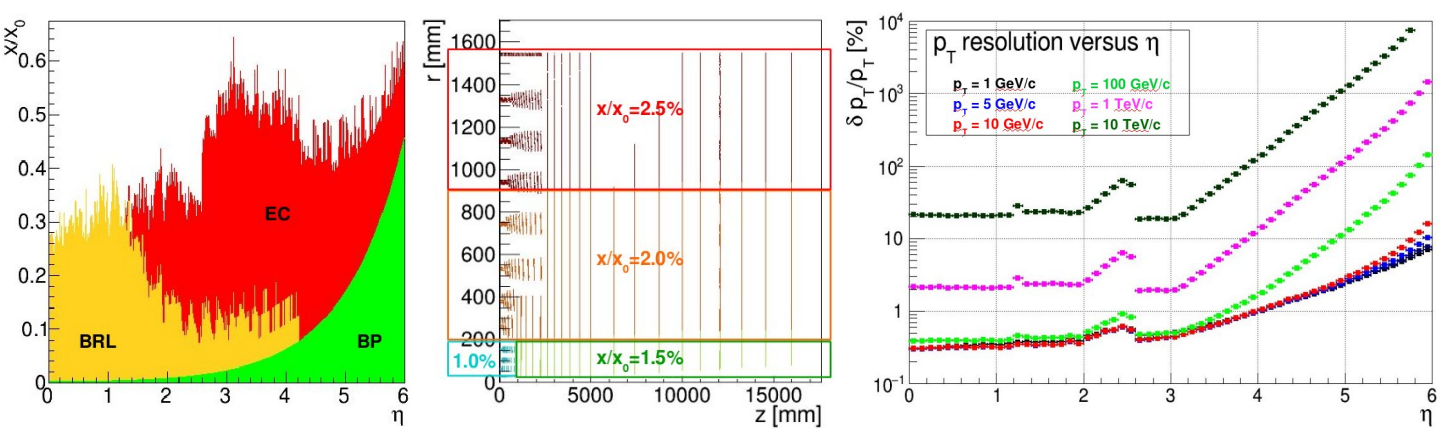

Figure 6: The tracker material distribution along $\eta$, in $x / x_{0}$. The beam-pipe is shown in green, BRL region in orange and EC region in red (left plot). Various regions of tracker are assigned a different material budget per layer, in percentage of $x_{0}$ : innermost vertex layers (1\%), innermost forward rings $(1.5 \%)$, a macro-pixel region $(2.0 \%)$ and an outer region $(2.5 \%)$ (middle plot). As an outcome, the anticipated $\Delta p_{\mathrm{T}} / p_{\mathrm{T}}$ resolution, as simulated by tkLayout $\mathrm{SW}$, is shown on the right plot for different $p_{\mathrm{T}}$ values: $1 \mathrm{GeV} / \mathrm{c}$ (black), $5 \mathrm{GeV} / \mathrm{c}$ (blue), $10 \mathrm{GeV} / \mathrm{c}$ (red), $100 \mathrm{GeV} / \mathrm{c}$ (light green), $1 \mathrm{TeV} / \mathrm{c}$ (magenta) and $10 \mathrm{TeV} / \mathrm{c}$ (dark green).

phase 2 tracker studies and has now been adapted (as tkLayout-lite) for FCC-hh needs [16]. More 
details on tracker performance, studied in various tracker configurations, may be found here [17].

\section{Pattern-recognition, Primary Vertexing and Pile-up Mitigation}

Pattern recognition is a very challenging mathematical problem and a key aspect in the overall tracker design. The goal is to optimize a granularity in $Z$, a distance between individual measurement layers and a suitable tilt of detector modules. To fully solve this problem, a full simulation framework (Pythia+Geant4) is needed, with a detailed geometry/material description and a dedicated track fitting/propagator SW package. To avoid such a complex approach, we have used simpler assumptions: a perfect seeding (non-trivial), simplified track parameters error propagator (tkLayout-lite), simplified geometry description (tkLayout-lite) and a knowledge of background hits distribution (Fluka [10]). Our method is based on a probabilistic approach and aims to qualitatively understand an optimal tracker design in a view of pattern recognition. Let us denote $p_{\text {bkg_95\%CL }}^{i}$ a probability, that at $i$-th layer/disc a track signal hit may be compromised by a background hit at $95 \%$ confidence level. The final product of individual probability complements, $\left(1-p_{\text {bkg_95\%CL }}^{i}\right)$, cumulated across all crossed tracker layers/rings, $i$, may be then approximatively referred as a pattern-recognition efficiency. The seeding problem is not considered within the study, hence we assume perfect seeding and sum up probability complements over all planes except the first 3 seeding layers/rings. In other words, we start from a perfect seed, propagate the track, apply an error ellipse approach to calculate a signal-background merge probability and finally, cumulate their complements as:

$$
\varepsilon \sim \prod_{i=4}^{N}\left(1-p_{\text {bkg_95\%CL }}^{i}\right)
$$

Such a method points out to the "weak" spots in the geometry layout for low $p_{\mathrm{T}}$ tracking. To

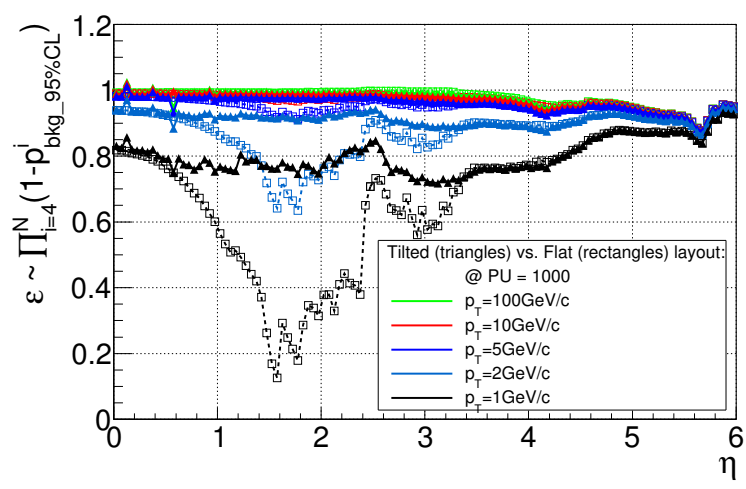

Figure 7: Pattern-recognition for a tracker layout in 2 variants: a traditional flat layout v3.03 [17] (rectangles, dashed), and a reference layout v4.01 (triangles, solid) with tilted modules in BRL/EC transition region.

clearly interpret its results, one needs to initially "calibrate" $\varepsilon$. The technique has been thus applied to a CMS phase 2 geometry v3.6.5 [16, 18] (PU=140). For this scenario full simulation results exist and tracks reconstruction seems feasible. This gives a limit estimate $\varepsilon=80 \%$ for $p_{\mathrm{T}}=1 \mathrm{GeV} / \mathrm{c}$. The value is used as a reference for our layout, but in FCC-hh pile-up=1000. As shown in Fig. 7, the tilted compared to traditional flat layout is a must, namely in the BRL/EC region $(0.5<|\eta|<2.5)$. The key is to minimize the material effect and the error ellipse projection factor in $Z$. 
The next important optimization aspect is the primary vertexing (for illustration see Fig. 4). To quantitatively understand the problem, the study of effective pile-up for low $p_{\mathrm{T}}$ tracks is suggested. By assuming that a signal vertex can be found by a dedicated algorithm from e.g. high $p_{\mathrm{T}}$ tracks, one may raise a question of how many pile-up vertices effectively lead to a confusing assignment of low $p_{\mathrm{T}}$ tracks to the original primary vertex. This quantity is called an effective pile-up and is studied in 2 scenarios: with and without track timing information. Without timing information, only the $\delta\left(z_{0}\right)$ can be used as a quality measure. With timing information, a 2D $\chi^{2}$ approach can be applied. To understand the impact of effective pile-up in more detail, this strategy has been initially applied to the CMS phase 2 geometry v3.6.5 and then to the FCC-hh. As depicted in Fig. 7, in CMS scenario at $|\eta|=4.0$ (by assuming $\delta t=25$ ps per track) there are effectively 1.2 pile-up vertices to be dealt with in average for $1 \mathrm{GeV} / \mathrm{c} p_{\mathrm{T}}$ tracks. As demonstrated by full simulations [18], such a value does not seem to be an issue for a high level reconstruction. For FCC-hh and low $p_{\mathrm{T}}$ tracks, the 1D vertexing will lead to an effective pile-up of tens or even hundreds for $|\eta|>3.0$ and hence, $2 \mathrm{D}$ vertexing with an extreme timing resolution $5-10 \mathrm{ps}$ per track seems essential. Yet, the primary vertexing beyond $|\eta|>4.0$ may be still challenging.

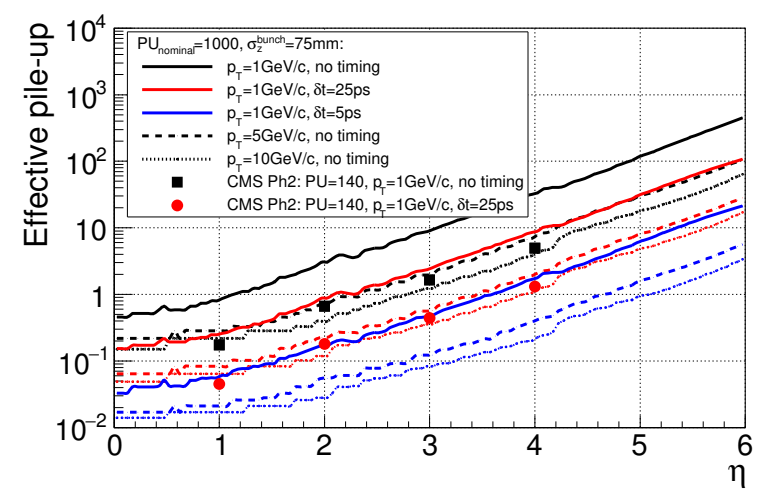

Figure 8: An effective pile-up for the tracker in tilted layout and nominal FCC-hh scenario. Several options of timing resolution per track in 2D vertexing are assumed: no timing (black), $\delta t=25 \mathrm{ps}$ (red) and $\delta t=5 \mathrm{ps}$ (blue). Several $p_{\mathrm{T}}$ values are shown: $1 \mathrm{GeV} / \mathrm{c}$ (solid), $5 \mathrm{GeV} / \mathrm{c}$ (dashed) and $10 \mathrm{GeV} / \mathrm{c}$ (dotted). For reference the effective pile-up for CMS Ph2 layout, $p_{\mathrm{T}}=1 \mathrm{GeV} / \mathrm{c}$ and nominal pile-up=140 is added.

\section{Summary and Outlook}

The FCC-hh represents a truly challenging project in all its aspects: an infrastructure, accelerator and corresponding detector system. The current effort in the FCC-hh detector working group focuses on the key challenges of such an environment with the main goal to produce a proved concept of the detector system. The first milestone for the study is to prepare a common CDR for the European Strategy Update by end of 2018. To fully exploit the current CERN expertise a strong emphasis is placed on connecting various experts across different LHC experiments, including their upgrades towards the HL-LHC and non-LHC projects (e.g. CLIC). Despite that, some FCC-hh challenges extend much beyond the current projects and therefore new conceptual ideas need to be invented and a dedicated R\&D set up. For the tracker, the key challenges are mainly an increased number of pile-up events $\mathrm{O}(1000)$ (compared to HL-LHC), extreme particle fluences and huge ra- 
diation levels. These all make the track pattern recognition, particle tracking, $b, c, \tau$-tagging and identification of vertices an extremely interesting, but also challenging task.

\section{References}

[1] N. Arkani-Hamed, et al., Physics opportunities of a $100 \mathrm{TeV}$ proton-proton collider Physics Reports, 652 (2016) p. 1-49 [https://doi.org/10.1016/j.physrep.2016.07.004]

[2] M. McCullough, Physics at FCC talk at FCC Week in Berlin (2017) [https://indico.cern.ch/event/556692/contributions/2483388]

[3] Future Circular Collider Project [https://fcc.web.cern.ch]

[4] CEPC-SPPC Study Group, CEPC-SPPC Preliminary Conceptual Design Report, Volume 2 IHEP-AC-2015-01 (2015) [http://inspirehep.net/record/1395736]

[5] The ATLAS Collaboration, The ATLAS Experiment at the CERN Large Hadron Collider JINST 3 S08003 (2008) [http://iopscience.iop.org/article/10.1088/1748-0221/3/08/S08003]

[6] The CMS Collaboration, The CMS experiment at the CERN LHC JINST 3 S08004 (2008) [http://iopscience.iop.org/article/10.1088/1748-0221/3/08/S08004]

[7] M. Benedikt, FCC: Study Status \& Plans talk at FCC Week in Berlin (2017) [https://indico.cern.ch/event/556692/contributions/2483390]

[8] W. Riegler, Summary of FCC-hh Experiments and Detector talk at FCC Week in Rome (2016) [https://indico.cern.ch/event/438866/contributions/1084924]

[9] G. Apollinari, et al., High-Luminosity Large Hadron Collider (HL-LHC) - Preliminary Design Report CERN Yellow Reports: Monographs, CERN-2015-005 (2015) [https://cds.cern.ch/record/2116337]

[10] M.I. Besana, F. Cerutti, et. al., Radiation Environment talk at FCC Week in Berlin (2017) [https://indico.cern.ch/event/556692/contributions/2465156]

[11] R. Contino, et. al., Physics at a $100 \mathrm{TeV}$ pp collider: Higgs and EW symmetry breaking studies [arXiv:1606.09408]

[12] W. Riegler, FCC-hh experiments \& detectors - CDR plan and status talk at FCC Week in Berlin (2017) [https://indico.cern.ch/event/556692/contributions/2483417]

[13] H.F. Pais Da Silva, et. al., FCC-hh Detector - Integration and Opening-Closing Scenarios talk at FCC Week in Berlin (2017) [https://indico.cern.ch/event/556692/contributions/2592515]

[14] H. ten Kate, M. Mentink, et. al., FCC-hh Detector Magnet - Evolution and New Baseline Design talk at FCC Week in Berlin (2017) [https://indico.cern.ch/event/556692/contributions/2465157]

[15] S. Fartoukh, Pile up management at the high-luminosity LHC and introduction to the crab-kissing concept

Phys. Rev. Accel. Beams 17, 111001 (2014) [http://dx.doi.org/10.1103/PhysRevSTAB.17.111001]

[16] CMS tkLayout working group tkLayout - an official CMS version [https://github.com/tkLayout/tkLayout] tkLayout-lite - an adapted version for the FCC-hh studies [https://github.com/drasal/tkLayout]

[17] FCC-hh Tracker - tkLayout performance web page [http://fcc-tklayout.web.cern.ch]

[18] The CMS Collaboration, The Phase-2 Upgrade of the CMS Tracker CERN-LHCC-2017-009 (2017) [http://cds.cern.ch/record/2272264] 